\title{
FACTORES PREDICTORES DEL SENTIDO DE VIDA EN ADULTOS MAYORES DEL SURESTE DE MÉXICO
}

\author{
Miguel Ángel Tuz-Sierra \\ Facultad de Humanidades. Universidad Autónoma de Campeche, México \\ migatuz@uacam.mx \\ Eric Alejando Catzín-López \\ Facultad de Humanidades. Universidad Autónoma de Campeche, México \\ ericalejandro95@gmail.com \\ Alejandra Kantun-Dzul \\ Facultad de Humanidades. Universidad Autónoma de Campeche, México \\ al045806@uacam.mx \\ Erika Rendon-Sosa \\ Facultad de Humanidades. Universidad Autónoma de Campeche, México \\ al044052@uacam.mx \\ Gabriela Isabel Pérez-Aranda \\ Facultad de Humanidades. Universidad Autónoma de Campeche, México \\ gaiperez@uacam.mx
}

Recepción Artículo: 26 marzo 2021 Admisión Evaluación: 26 marzo 2021 Informe Evaluador 1: 27 marzo 2021 Informe Evaluador 2: 29 marzo 2021 Aprobación Publicación: 30 marzo 2021

\section{RESUMEN}

El Sentido de Vida es una variable asociada directamente a la salud mental; debido al rápido envejecimiento de la población y el alargamiento de la esperanza de vida, es necesario realizar investigaciones que tengan como punto central al adulto mayor. En este sentido se estudió una muestra no probabilística de 540 adultos mayores, de los cuales 200 residen en asilo público, 120 en asilo privado, 120 con algún familiar y 100 viven solos. Se trató de un estudio no experimental, transversal y comparativo. La variable sentida de vida se midió a través del test PIL (Purpose in life). Se encontró que el $61.1 \%$ de la muestra carecen de Sentido de Vida, existiendo diferencias estadísticamente significativas $(p<0.05)$ entre los grupos, siendo quienes residen con algún familiar los que presentan la media más alta (119.50), mientras que aquellos que residen en asilo público presentan la media más baja (78.16). Se realizó un modelo de regresión lineal múltiple donde resultó que los factores que más influyen en el Sentido de Vida de la muestra son Percepción de la situación familiar, Espiritualidad y religión, así como Percepción de la salud.

Palabras clave: sentido de vida; adultos mayores; envejecimiento; familia 


\section{FACTORES PREDICTORES DEL SENTIDO DE VIDA EN ADULTOS MAYORES DEL SURESTE DE MÉXICO}

\section{ABSTRACT}

Purpose in life predictive factors of older adults in mexican southeast. The purpose of life is a variable directly associated with mental health; Due to the rapid aging of the population and the lengthening of life expectancy, it is necessary to carry out research that focuses on the elderly. In this sense, a non-probabilistic sample of 540 older adults was studied, of which 200 reside in public asylum, 120 in private asylum, 120 with a family member and 100 live alone. It was a nonexperimental, cross-sectional and comparative study. It was found that $61.1 \%$ of the sample does not have a sense of purpose in life, there are statistically significant differences $(p<0.05)$ between the groups, being those who reside with a family member who have the highest mean of this variable (119.50), while those residing in public asylum have the lowest average (78.16). A multiple linear regression model was carried out where it turned out that the factors that most influence the Sense of Life of the sample are Perception of the family situation, Spirituality and religion, as well as Perception of health.

Keywords: purpouse of life; elders; aging; family

\section{INTRODUCCIÓN}

La población adulta mayor, personas de 65 años o más, va incrementando de manera acelerada en todos los países del mundo, sin importar el nivel de desarrollo de estos; según datos de la OMS (2015) entre 2000 y 2050, la proporción de los habitantes del planeta mayores de 60 años se duplicará, pasando del 11\% al 22\%. En México, para el último censo poblacional en el 2015, el 7.3\% de la población era adulta mayor, es decir, 8, 755, 508 personas en todo el país se encontraban en una edad de 65 años o más (INEGI, 2015), la tendencia indica que las cifras de personas de 65 años 0 más seguirá en aumento. Para el Estado de Campeche estas cifras se traducen, según datos del INEGI (2015) en el 6.5\% de la población, lo que representa 58646 personas, que tienen una edad de 65 años 0 más.

El aumento de la esperanza de vida y los avances médicos y tecnológicos, han contribuido con el aumento de la población adulta; al hacer una revisión de las condiciones de vida de los adultos mayores, en su mayoría desfavorables, cobra especial relevancia atender urgente y oportunamente los problemas relacionados a la salud. Pero no solo la salud física resulta crucial para la adultez mayor, la salud mental toma un papel importante para un desarrollo exitoso.

Tener un sentido de vida es un referente psicológico a que cada circunstancia puede convertirse en una razón por la cual mantener un propósito, en otras palabras, indica que los problemas pueden volverse oportunidades para darle significado a la existencia (Frankl, 2004). En este último aspecto, el sentido de vida cobra especial importancia en la adultez mayor, sobre todo después del retiro situación en la que muchas personas pueden dejar de sentirse útiles y productivas; sin embargo, aunque en este momento de la vida suele ser donde se recapitulen las experiencias de cada persona, este sentido en lugar de perderse, podría tener una oportunidad de transformarse, y esto es posible cuando se recurre a los propios recursos y a la resignificación de la historia de nuestra propia vida.

En el campo de la salud mental existe una carencia de atención debidamente orientada a la solución de sus problemas, los cuales se asocian con un origen multifactorial donde los factores personales y sociales confluyen desfavorablemente. Específicamente, el individuo que se ve afectado por problemas de salud mental tales como ansiedad, depresión, alcoholismo, etc., ve disminuido su bienestar y funcionalidad, lo que se refleja en su calidad de vida en general. Estas afectaciones pueden variar de persona a persona ya que cada individuo vive y percibe sus experiencias y su entorno de manera diferente; lo cual puede cambiar a lo largo del ciclo vital (Ornelas \& Ruíz, 2017).

Hoy en día, temas como la depresión y el aumento del número de casos de suicidio son un tema 
de salud pública que afecta a todos los grupos de edad, sin embargo, la adultez mayor se posiciona como una de las edades más sobresalientes en este aspecto. Según datos del Instituto Mexicano del Seguro Social (2014) la depresión es una enfermedad mental que afecta a más de 350 millones de personas en todo el mundo, siendo los Adultos Mayores uno de los grupos más vulnerables.

Autores como, Sotelo-Alonso et al (2012) mencionan que la depresión se considera un problema de salud pública, con graves implicaciones en la discapacidad, la morbilidad, la mortalidad, así como la calidad de vida de los ancianos que la padecen (...) en cuanto al suicidio como complicación de la depresión, se observa que su tasa se correlaciona en forma directa con la edad, siendo en promedio de 2.6/100 000, más alta de lo reportado en otras épocas de la vida.

Ciertamente, en cuanto a los suicidios en México, los actores y víctimas principales son los jóvenes entre 15 y 34 años, también se encuentran personas mayores de 65 años, en su inmensa mayoría del sexo masculino. Ambos grupos de edad son, por el cambio demográfico debido al envejecimiento de la población, cada vez más numerosos, por lo que el número de suicidios seguirá probablemente aumentando si se conservan las actuales condiciones socioeconómicas y la falta de medidas preventivas .

De igual manera existe una relación entre el inicio de la depresión y las pérdidas en la vejez. Estas pérdidas que ya se inician en la edad madura están asociadas por ejemplo a la melancolía involutiva, se acentúan a medida que avanza la edad. Estas pérdidas son las del propio cuerpo, a la de los roles sociales y familiares, a la del status socioeconómico y en última instancia a la anticipación de la propia muerte. Todas estas situaciones podrían favorecer la pérdida del sentido de vida en los adultos mayores.

Uno de los factores que pudieran contribuir a la depresión y al suicidio en los adultos mayores, es la pérdida del sentido de vida, es decir, el no tener un motivo o propósito claro para continuar viviendo, esta pérdida de sentido esta, por lo general, ligada a un conjunto de situaciones tales como la pérdida de la capacidad física, cognitiva, abandono por parte de la familia y pérdida de familiares 0 seres queridos, entre otros. Es por ello por lo que resulta esencial continuar brindando atención a los adultos mayores, pero no solo en el ámbito médico, sino también desde el ámbito psicológico y espiritual

Actualmente, en México, gran parte de la investigación realizada se ha enfocado al estudio y desarrollo de programas para niños y adolescentes, en realidad, son muy reducidos los estudios realizados con adultos mayores y aún menor el número de investigaciones que se plantean estudiar aspectos psicológicos-filosóficos como el sentido de vida. Prestar especial atención al bienestar de los grupos vulnerables, abarca un punto importante en la investigación, para aportar recursos que contribuyan al cuidado de la salud mental, incluyendo la prevención e intervención.

No obstante, debido al incremento de la población de esta edad y a las proyecciones a futuro para el país, es necesario generar conocimiento sobre la adultez mayor en aras de desarrollar estrategias, programas y políticas públicas que favorezcan el bienestar, tanto físico, como psicológico y social de la población que se encuentra en esta etapa de la vida, así como para la asistencia en la búsqueda del sentido de la vida que resulta crucial en esta etapa. Tomando en cuenta lo anterior, existe un factor que influye sin importar el grupo de edad, y este es: el género; hay una brecha entre el ser mujer y hombre, significa experimentar las etapas y papeles en la sociedad de diferente manera, y por ello surge la necesidad de investigar en que factores difieren estos roles y que, por tanto, impacta en el sentido de la vida de cada persona, incluso en cómo pueden llegar a perderlo. 


\section{FACTORES PREDICTORES DEL SENTIDO DE VIDA EN ADULTOS MAYORES DEL SURESTE DE MÉXICO}

\section{MÉTODO}

\section{Diseño}

Se utilizó un diseño no experimental, transversal; se trató de un estudio empírico con metodología cuantitativa haciendo uso de un diseño ex post facto, específicamente en la modalidad de estudio retrospectivo simple debido a que se eligió un grupo clave que comparte una característica (ser adultos mayores), se estudió una variable dependiente (sentido de vida) y posteriormente se analizó su relación con otras variables incluyendo algunas posibles variables independientes.

\section{Participantes}

La muestra fue no probabilística compuesta y por estratos conformada por 540 participantes adultos mayores sin problemas neurocognitivos que residen en la ciudad de San Francisco de Campeche, Campeche, en el sureste de México; de los cuales 200 residen en asilo público, 120 en un asilo privado, 120 con algún familiar y 100 viven solos.

\section{Instrumentos}

El instrumento que se utilizó para medir la variable Sentido de Vida fue la prueba PIL (Purpose in life), es una escala de aptitudes construida a partir de la orientación de la logoterapia; la escala tiene la finalidad de medir el concepto básico de Frankl conocido como "vacío existencial". La prueba conformada por 3 partes, la parte A son 20 enunciados que se responden en escala Likert, la parte $B$ se constituye por 13 frases incompletas y por último la parte $C$ es de tipo abierta en donde el sujeto describe sus metas, ambiciones y deseos en la vida según la medida en los que los alcanzo o los está alcanzando. Se analizó la confiabilidad del instrumento mediante Alfa de Cronbach obteniendo una confiabilidad de .937.

De igual forma, para conocer e identificar algunas de las características del adulto y adulta mayor, se utilizó una encuesta de datos generales que consta de 28 ítems, conformada por 5 dimensiones: factores situacionales y de salud física/mental; espiritualidad y religión; percepción de situación económica; percepción de situación familiar y; estilo de residencia.

La confiabilidad de cada factor fue analizada mediante Alfa de Cronbach; en donde se obtuvieron los siguientes resultados: percepción de salud mental y física obtuvieron .762. Espiritualidad y religión obtuvieron .801, por otro lado, situación económica tiene un puntaje de .962, así como situación familiar con .977.

\section{Procedimiento}

Para la recolección de los datos se contó con una única versión a lápiz y papel, previo a la administración de estos se solicitó la firma de una carta de consentimiento informado en donde se explicó de manera detallada la confidencialidad de la información recabada. Los datos fueron procesados y analizados por medio del Paquete Estadístico para las Ciencias Sociales (SPSS) en su versión 23.

\section{RESULTADOS}

En primer lugar, se analizaron los datos descriptivos; el análisis se realizó de los cuatro factores situacionales estudiados: Percepción de la salud; Espiritualidad y religión; Percepción de la situación familiar y; Percepción de la Situación Económica. Así como del Sentido de Vida (medido a través del PIL), y la percepción que los adultos mayores tienen del trato que se le da en el lugar donde reside. Posteriormente se realizó un análisis de correlaciones, así como análisis de comparaciones de medias mediante la prueba t y ANOVA. 
En general los adultos mayores se ubicaron en niveles altos en todos los factores situacionales (Tabla 1), resaltando el factor Espiritualidad y religión, así como Percepción de la situación familiar. Respecto al factor Percepción de situación económica, a pesar de que la mayor parte de la muestra se ubicó en el nivel alto, una parte considerable (33.3\%) se ubicó en el nivel bajo.

Tabla 1

Frecuencia en Porcentaje de los Factores Situacionales de los Adultos Mayores

\begin{tabular}{ccccc}
\hline Categoría & $\begin{array}{c}\text { Percepción de } \\
\text { la salud }\end{array}$ & $\begin{array}{c}\text { Espiritualidad y } \\
\text { religión }\end{array}$ & $\begin{array}{c}\text { Percepción de } \\
\text { situación familiar }\end{array}$ & $\begin{array}{c}\text { Percepción de } \\
\text { situación } \\
\text { económica }\end{array}$ \\
\hline Bajo & $11.1 \%$ & $9.3 \%$ & $20.4 \%$ & $33.3 \%$ \\
Medio & $33.3 \%$ & $20.4 \%$ & $14.8 \%$ & $24.1 \%$ \\
Alto & $55.6 \%$ & $70.4 \%$ & $64.8 \%$ & $42.6 \%$ \\
\hline
\end{tabular}

Respecto al factor Percepción del trato en el estilo de residencia (Tabla 2), un 64.8\% de los adultos mayores se ubicaron en el nivel alto, refiriendo así percibir un trato adecuado en el lugar donde residen, no obstante, es importante recalcar que un $35.2 \%$ de la muestra se ubicó entre el nivel medio y bajo de percepción del trato.

Tabla 2

Percepción del Trato en el Estilo de Residencia de los Adultos Mayores

\begin{tabular}{ccc}
\hline Categoría & Frecuencia & Porcentaje \\
\hline Bajo & 120 & 22.2 \\
Medio & 70 & 13.0 \\
Alto & 350 & 64.8 \\
\hline
\end{tabular}

En cuanto a la variable central del estudio, el Sentido de vida (Tabla 3), la muestra se ubicó equilibrada entre las distintas categorías, de forma tal que un 33.3\% ubicó en la categoría carencia de sentido, un $27.8 \%$ en la categoría Indefinición de sentido, y un $38.9 \%$ en la categoría Presencia de sentido. Un punto relevante es que, en conjunto, un $61.1 \%$ de los participantes se ubican entre la carencia y la indefinición de sentido, lo que indica que esta parte de la muestra no cuenta con un sentido de vida definido.

Tabla 3

Sentido de Vida de los Adultos Mayores

\begin{tabular}{lcc}
\hline Categoría & Frecuencia & Porcentaje \\
\hline Carente de sentido & 180 & 33.3 \\
Indefinición de sentido & 150 & 27.8 \\
Presencia de sentido & 210 & 38.9 \\
\hline
\end{tabular}

El análisis de correlaciones mediante la prueba $r$ de Pearson, arrojó diversos datos estadísticamente significativos $(p<0.05)$, mismos que se muestran en la tabla 4 . Dentro de los datos más relevantes se puede mencionar la relación positiva y sustancial entre la Percepción de la situación económica y la Percepción de la situación familiar, indicando que aquellos sujetos que se perciben con buenas relaciones familiares tienden a percibir de mejor manera su economía. Así mismo, se encontró una correlación positiva y marcada entre la Percepción de la situación familiar y la Percepción del trato en el estilo de residencia, lo cual refiere que los sujetos que perciben su situación familiar como adecuada, tienden a percibir mejor el trato en el lugar donde residen. La variable Sentido de 


\section{FACTORES PREDICTORES DEL SENTIDO DE VIDA EN ADULTOS MAYORES DEL SURESTE DE MÉXICO}

vida se correlacionó de manera positiva y sustancial con todos los factores situacionales evaluados, encontrándose la correlación más fuerte con el factor Percepción de la situación familiar. De la misma manera, se encontró una correlación positiva leve entre el Sentido de vida y la Percepción del trato en el estilo de residencia indicando que aquellos individuos que perciben de mejor manera el trato que reciben en el lugar donde viven, tienen a presentar índices más altos de sentido de vida.

De manera general, es importante mencionar la relevancia que el factor Percepción de la situación familiar tuvo en el análisis correlacional, lo que realza la importancia de esta institución social en la vida de los adultos mayores.

Tabla 4

Correlaciones de Factores Situacionales y Sentido de Vida

\begin{tabular}{|c|c|c|c|c|c|c|}
\hline & & $\begin{array}{l}\text { Percepción } \\
\text { de la salud }\end{array}$ & $\begin{array}{c}\text { Espiritualidad } \\
\text { y religión }\end{array}$ & $\begin{array}{c}\text { Percepción } \\
\text { de la } \\
\text { situación } \\
\text { económica }\end{array}$ & $\begin{array}{l}\text { Percepción } \\
\text { de la } \\
\text { situación } \\
\text { familiar }\end{array}$ & $\begin{array}{c}\text { Percepción } \\
\text { del trato } \\
\text { en el estilo } \\
\text { de } \\
\text { residencia }\end{array}$ \\
\hline \multirow{2}{*}{$\begin{array}{l}\text { Sentido de } \\
\text { Vida }\end{array}$} & $\mathrm{r}$ & $.426^{*}$ & $.450^{*}$ & $.404^{*}$ & $.475^{*}$ & $.373^{*}$ \\
\hline & p & .001 & .001 & .002 & .000 & .006 \\
\hline \multirow{2}{*}{$\begin{array}{l}\text { Percepción } \\
\text { de la salud }\end{array}$} & $\mathrm{r}$ & & & & & $.332^{*}$ \\
\hline & $\mathrm{p}$ & & & & & .014 \\
\hline \multirow{2}{*}{$\begin{array}{c}\text { Espiritualidad } \\
\text { y religión }\end{array}$} & $\mathrm{r}$ & & & & $.306^{*}$ & $.331^{*}$ \\
\hline & $\mathrm{p}$ & & & & .024 & .014 \\
\hline \multirow{2}{*}{$\begin{array}{c}\text { Percepción } \\
\text { de la } \\
\text { situación } \\
\text { económica }\end{array}$} & $r$ & & & & $.524^{*}$ & $.396^{*}$ \\
\hline & $\mathrm{p}$ & & & & .000 & .003 \\
\hline \multirow{2}{*}{$\begin{array}{l}\text { Percepción } \\
\text { de la } \\
\text { situación } \\
\text { familiar }\end{array}$} & $\mathrm{r}$ & & & & & $.614^{*}$ \\
\hline & $\mathrm{p}$ & & & & & .000 \\
\hline
\end{tabular}

* Dato estadísticamente significativo $(\mathrm{p}<.05)$

Se analizaron las medias obtenidas referente al sentido de vida según el estilo de residencia de los sujetos (Tabla 5) y se encontraron diferencias estadísticamente significativas $(p<0.05)$ entre los diversos grupos de estilo de residencia, siendo las personas que viven con un familiar quienes obtuvieron la media más alta, seguidos de aquellos quienes viven solos, aquellos quienes viven en un asilo público y, por último, las personas que obtuvieron una media menor de Sentido de vida fueron quienes viven en asilo privado.

Tabla 5

Comparación de Medias del Sentido de Vida según el Estilo de Residencia

\begin{tabular}{lccccc}
\hline \multicolumn{1}{c}{ Categoría } & $\mathrm{N}$ & Media & Desviación estándar & $\mathrm{F}$ & $\mathrm{P}$ \\
\hline Asilo público & 200 & 102.60 & 24.04 & & \\
Asilo privado & 120 & 78.16 & 35.71 & 4.41 & $.008^{*}$ \\
Con familiar & 100 & 119.50 & 14.92 & & \\
Sólo & 120 & 104.33 & 30.41 & & \\
\hline
\end{tabular}

* Dato estadísticamente significativo 
Respecto al análisis del Sentido de vida según el estilo de residencia, se encontraron diferencias estadísticamente significativas entre estos grupos $(p<0.05)$, en donde las personas que residen con un familiar perciben recibir un mejor trato en el lugar donde residen, seguidos de las personas que residen solos, las personas que residen en asilo público y, por último, las personas que perciben un trato menos favorable en su lugar de residencia fueron aquellos quienes residen en asilo privado.

Tabla 6

Comparación de Medias de Percepción del Trato Según el Estilo de Residencia

\begin{tabular}{lccccc}
\hline \multicolumn{1}{c}{ Categoría } & $\mathrm{N}$ & Media & Desviación estándar & $\mathrm{F}$ & $\mathrm{P}$ \\
\hline Asilo público & 200 & 11.35 & 5.019 & & \\
Asilo privado & 120 & 9.00 & 4.918 & 3.876 & $.014^{*}$ \\
Con familiar & 100 & 14.90 & .316 & & \\
Sólo & 120 & 12.75 & 3.519 & & \\
\hline
\end{tabular}

* Dato estadísticamente significativo

Se realizó también un análisis de comparación de medias del Sentido de Vida según el sexo de los participantes, en donde se obtuvo que los hombres presentan una media de 104.95 mientras que las mujeres presentan una media de 97.26 , es decir, los hombres presentan una media 7.69 puntos por encima que las mujeres, sin embargo, tras el análisis con la prueba ANOVA, no se encontraron diferencias estadísticamente significativas entre los grupos.

Tabla 7

Comparación de Medias de Sentido de Vida por Sexo

\begin{tabular}{cccccc}
\hline & Sexo & N & Media & F & P \\
\hline \multirow{2}{*}{ Sentido de vida } & Hombre & 240 & 104.95 & \multirow{2}{*}{9.78} & \multirow{2}{*}{.333} \\
& Mujer & 300 & 97.26 & & \\
\hline
\end{tabular}

Por último, se aplicó un modelo de regresión lineal múltiple con la finalidad de encontrar que elementos explican mejor el Sentido de vida de los adultos mayores. El modelo generado obtuvo un $\mathrm{R}$ cuadrado ajustado de .516, lo que indica que dicho modelo explica la varianza del Sentido de Vida en un $51.6 \%$, el modelo resultó ser estadísticamente significativo $(p<0.05)$.

Tabla 8

Resumen del Modelo de Regresión lineal Múltiple

\begin{tabular}{ccccccc}
\hline Modelo & $\mathrm{R}$ & $\mathrm{R}$ & $\begin{array}{c}\text { R cuadrado } \\
\text { ajustado }\end{array}$ & $\begin{array}{c}\text { Error estándar } \\
\text { de la } \\
\text { estimación }\end{array}$ & $\begin{array}{c}\text { Estadísticos de cambio } \\
\text { Cambio en } \\
\text { R cuadrado }\end{array}$ & $\begin{array}{c}\text { Sig. } \\
\text { Cambio } \\
\text { en } \mathrm{F}\end{array}$ \\
\hline 1 & $.750^{\mathrm{a}}$ & .562 & .516 & 20.72 & .562 & $.000^{*}$ \\
\hline
\end{tabular}

Al analizar la viabilidad del modelo de regresión con la prueba ANOVA, se encontró un valor $\mathrm{P}$ de .000 , lo que indica que, en efecto, el modelo es viable para explicar la variable dependiente. 


\section{FACTORES PREDICTORES DEL SENTIDO DE VIDA EN ADULTOS MAYORES DEL SURESTE DE MÉXICO}

Tabla 9

Viabilidad del Modelo de Regresión Lineal Múltiple con prueba ANOVA

\begin{tabular}{ccccccc}
\hline \multicolumn{2}{c}{ Modelo } & $\begin{array}{c}\text { Suma de } \\
\text { cuadrados }\end{array}$ & gl & $\begin{array}{c}\text { Media } \\
\text { cuadrática }\end{array}$ & F & Sig. \\
\hline 1 & Regresión & 26438.46 & 5 & 5287.69 & & \\
& Residuo & 20611.18 & 548 & 429.40 & 12.31 & $.000^{\mathrm{b}}$ \\
& Total & 47049.64 & 553 & & & \\
\hline
\end{tabular}

En general, de las variables introducidas en la ecuación como posibles variables independientes que explicaran el Sentido de vida, solo la Percepción de la situación familiar, la Espiritualidad y religión, así como la Percepción de la salud permiten explicar la variable en un $51.6 \%$ de su varianza. El factor que resulta influir más en el Sentido de vida es la Percepción de la situación familiar $(B=2.197)$. Las variables Percepción del estilo de residencia y Percepción de la situación económica no resultaron estadísticamente significativas $(p>0.05)$, por lo que no son consideradas como útiles para explicar el fenómeno en cuestión.

Tabla 10

Variables en la Ecuación

\begin{tabular}{|c|c|c|c|c|c|}
\hline \multirow{2}{*}{ Modelo } & \multicolumn{2}{|c|}{$\begin{array}{l}\text { Coeficientes no } \\
\text { estandarizados }\end{array}$} & \multirow{2}{*}{$\begin{array}{c}\text { Coeficientes } \\
\text { estandarizados }\end{array}$} & \multirow{2}{*}{$\mathrm{t}$} & \multirow{2}{*}{ Sig. } \\
\hline & B & $\begin{array}{l}\text { Desv. } \\
\text { Error }\end{array}$ & & & \\
\hline (Constante) & -16.845 & 15.789 & & -1.067 & .291 \\
\hline $\begin{array}{l}\text { Percepción del estilo } \\
\text { de residencia }\end{array}$ & -1.464 & .892 & -.223 & -1.642 & .107 \\
\hline $\begin{array}{l}\text { Percepción de la } \\
\text { situación familiar }\end{array}$ & 2.197 & .906 & .340 & 2.426 & $.019^{*}$ \\
\hline $\begin{array}{l}\text { Percepción de la } \\
\text { situación económica }\end{array}$ & 1.891 & .971 & .232 & 1.948 & .057 \\
\hline $\begin{array}{l}\text { Espiritualidad y } \\
\text { religión }\end{array}$ & 3.115 & .753 & .439 & 4.134 & $.000^{*}$ \\
\hline $\begin{array}{l}\text { Percepción de la } \\
\text { salud }\end{array}$ & 2.445 & .577 & .451 & 4.238 & $.000^{*}$ \\
\hline
\end{tabular}

\section{DISCUSIÓN Y CONCLUSIONES}

Después del análisis de los resultados obtenidos en la investigación, se encontró que la gran parte de los participantes se encuentran en un nivel alto de la percepción del trato que reciben en el lugar donde residen (64.8\%); respecto a esto, el INMUJERES menciona que tanto mujeres como hombres están expuestos a sufrir maltrato, sin embargo en México no se cuenta con la información que permita dimensionar la magnitud de este problema. No obstante, menciona que el $16 \%$ de los adultos mayores en México sufren rasgos de abandono y maltrato mientras que el $20 \%$ vive en soledad siendo el aislamiento del adulto mayor cada vez más frecuente en la sociedad mexicana. Diversos estudios constatan que el maltrato dirigido a los adultos mayores es el resultado de la combinación de factores personales, familiares, sociales, estructurales y culturales, por lo que la causa de este fenómeno es multidimensional y multifactorial .

De igual forma, se encontró que, en suma, un $61.1 \%$ de los participantes se encuentran en nive- 
les que sugieren carencia de sentido e indefinición de este, mientras que el 38.9\% presenta un sentido de vida definido, relacionado a esto, Jiménez \& Arguedas encontraron que los adultos mayores que tienen sentido de vida definido, lo tienen en función del servicio y transmisión de valores a otras generaciones en el caso de las mujeres y enseñar a otros en el caso de los hombres.

De igual manera se encontraron relaciones significativas del sentido de vida con la Percepción de la salud, la Espiritualidad y religión, la Percepción de la situación económica y la Percepción de la situación familiar siendo esta última la que representa la correlación más fuerte con el Sentido de Vida, esto se puede explicar a través de lo expuesto por el Instituto Internacional de Estudios sobre la Familia (The family watch) quien refiere que la familia tiene un papel positivo de las redes familiares, sociales y comunitarias en el bienestar general y la salud de los adultos mayores, como parte del envejecimiento activo que corresponde a la alta participación en un ámbito social. En un estudio realizado por Meléndez-Moral, Tomás-Miguel \& Navarro-Pardo encontraron que, aunque con diferencias en función del tipo, es la familia cercana, específicamente el o la cónyuge y los hijos, quienes brindan apoyo a los adultos mayores.

Así mismo, se encontraron diferencias significativas en el sentido de vida según el estilo de residencia de los sujetos, siendo las personas que viven en asilos privados quienes tienen menor presencia de sentido de vida, mientras que las personas que viven con algún familiar son quienes obtuvieron puntajes más elevados en el instrumento utilizado, esto concuerda con lo expuesto por Aguirre quien encontró en su investigación que las personas que viven en casas de reposo manifiestan bajos niveles de sentido de vida, mientras que aquellas que viven con algún familiar tienen mayor presencia de sentido.

La población adulta mayor representa un porcentaje cada vez más alto en la población mundial y México no es la excepción, es por ello que resulta fundamental incrementar estudios acerca de la salud del adulto mayor, tanto física como mental; en este sentido, el Sentido de Vida es un factor que funciona como indicador para tener una visión general de la salud mental de un sujeto, dado que la falta de este sentido se encuentra estrechamente relacionada a problemáticas mentales tales como la depresión; físicas como la pérdida de la capacidad cognitiva e inclusive sociales como abandono por parte de la familia.

Los resultados de la investigación permiten visualizar las importantes relaciones de algunos factores situacionales tales como la percepción de la situación familiar, económica y de salud, así como el lugar de residencia con el sentido de vida de los adultos mayores. Es importante mencionar que, con base en esta impresión diagnóstica de la situación problema, es posible generar algunas alternativas para atender las áreas de oportunidad que se identifican a través de los resultados, por ejemplo, la generación de políticas públicas que garanticen el bienestar de los adultos mayores, garantizando su no abandono, así como la implementación de programas sociales encaminados a incrementar las redes de apoyo sociales, familiares e institucionales de este sector de la población. Se considera relevante también, el monitorear y regular el trato y atenciones que ofrecen las instituciones que fungen como asilo para las personas adultas mayores.

A un nivel académico-científico, es importante el incremento de estudios relacionados a aspectos de la salud de esta población, pues es sólo a través de la visualización de la problemática como se podrá atender de mejor manera las necesidades.

\section{REFERENCIAS BIBLIOGRÁFICAS}

Aguirre, S. (2005). Manifestación del sentido de vida en personas de la tercera edad no institucionalizadas. Ajayu Órgano de Difusión Científica del Departamento de Psicología UCBSP.

Frankl, V. (2004). El hombre en busca del sentido. España: Herder. 


\section{FACTORES PREDICTORES DEL SENTIDO DE VIDA EN ADULTOS MAYORES DEL SURESTE DE MÉXICO}

Giraldo, L. (2016). El maltrato hacia las personas adultas mayores: una mirada desde la perspectiva de género. Obtenido de DIF: http://sitios.dif.gob.mx/cenddif/wpcontent/Archivos/BibliotecaDigital/EIMaltratoPersonasAdultas.pdf

Hernandez-Bringas, H., \& Flores-Arenales, R. (Junio de 2011). El suicidio en México. Obtenido de SCIELO: http://www.scielo.org.mx/scielo.php?script=sci_arttext\&pid=S140574252011000200004

INEGI. (2015). México en cifras. Obtenido de INEGI: http://www.beta.inegi.org.mx/app/areasgeograficas/

INEGI. (2015). Banco de indicadores. Obtenido de INEGI: http://www.beta.inegi.org.mx/app/indicadores/\#divFV1002000008

INMUJERES. (3 de junio de 2015). Situación de las personas adultas mayores en México. Obtenido de INMUJERES.gob: http://cedoc.inmujeres.gob.mx/documentos_download/101243_1.pdf

Instituto Internacional de Estudios sobre la Familia. (2013). El papel de la familia en el envejecimiento activo. Madrid: The Family Watch.

Instituto Mexicano del Seguro Social. (2014). Depresión. Obtenido de IMSS: http://www.imss.gob.mx/salud-en-linea/depresion

Jarne, A., Talarn, A., Armoyones, M., Horta, E., \& Requena, E. (2006). Psicopatología. España: Eureca Media.

Jiménez, F., \& Arguedas, I. (2004). Rasgos de sentido de vida del enfoque de resiliencia en personas mayores entre los 65 y 75 años. Actualidades Investigativas en Educación, 0.

Meléndez-Moral, J., Tomás-Miguel, J., \& Navarro-Pardo, E. (2007). Análisis de las redes sociales en la vejez a través de la entrevista Manheim. Salud Pública de México, 408-413.

OMS. (2015). Envejecimiento y ciclo de vida. Obtenido de Datos interesantes acerca del envejecimiento: http://www.who.int/ageing/about/facts/es/

Ornelas Ramírez, A., \& Ruíz Martínez, A. 0. (2017). Salud mental y calidad de vida: Su relación en Ios grupos etarios. PSIENCIA. Revista Latinoamericana de Ciencia Psicológica, 9. doi: 10.5872/psiencia/9.2.21 http://www.psiencia.org/9/2/21

Sotelo-Alonso, I., Rojas-Soto, J., Sánchez-Arena, C., \& Irigoyen-Coria, A. (marzo de 2012). La depresión en el adulto mayor: una perspectiva clínica y epidemiológica desde el primer nivel de atención. Obtenido de Redalyc: http://www.redalyc.org/pdf/507/50730962002.pdf

Romero, L. (26 de junio de 2017). ABANDONO Y MALTRATO DE ADULTOS MAYORES. Obtenido de Gaceta Digital UNAM: http://www.gaceta.unam.mx/20170626/abandono-y-maltrato-de-adultosmayores/ 